\title{
Postural deficiency syndrome, psychological evaluation
}

\author{
Pierre LECOMTE, Angélique JORAND
}

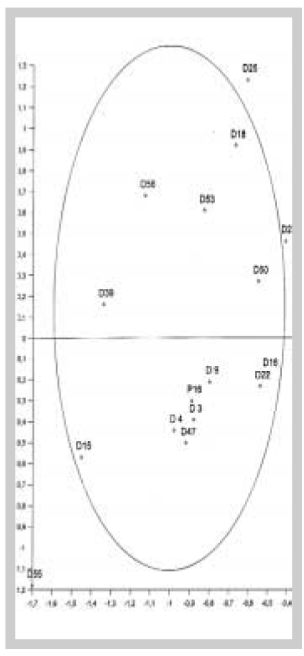

\section{ABSTRACT}

The purpose of this study is about the psychological suffering of the patients suffering from Postural Deficiency Syndrome. Our objective is to define this psychological suffering and find the most adapted tool to highlight this pain that would be easy to use by all practitioners interested in this population. 18 patients suffering from Postural Deficiency Syndrome (PDS) were compared to a reference population, comprising 55 chronic pain patients (for more than 2 months). The main evaluation criteria "Anxiety Depression Scale" concerns items in relation with slight anxiety or depression. Secondary criteria of this study are: Visual Analogical Scales (VAS, measuring pain intensity), Pain Sites Scoring (topographic scale), DALLAS multidimensional questionnaire (functional scale) and abridgement Beck Questionnaire (evaluation of depression). We use a multidimensional statistical evaluation (factorial analysis of correspondence) and the khi2 test.

The Anxiety Depression Scale analysis separate two subpopulations: "anxious or depressed" and "not anxious and not depressed". There is no significant difference between the postural patients and de reference population. The two populations present the same psychological disease. The patients suffering from chronic pain expressed much higher pain than the postural patients, at the VAS analysis. The visual topographic pain scale does not contribute to differentiate the populations. The DALLAS functional scale analysis reveals the small infirmity of postural patients on usual items of chronic pain. BECK questionnaire analysis does not show significant differences between chronic pain and postural patients. So the two populations present the same psychological suffering. The Anxiety Depression Score, main criteria of our study, is very well correlated to the Beck score and can be used alone for PDS patients. It helps non psychologists to distinguish PDS patients requiring adapted treatment. Secondary psychological pain with chronic symptoms indeed acts as a break in the medical care of PDS patients.

\section{KEYWORDS}

Psychological suffering

Address for correspondence:

Postural deficiency syndrome

P. LECOMTE,

Centre d'évaluation

et de traitement

de la douleur,

Chronic pain syndrome

Anxiety depression.

Hôtel-Dieu,

CS 26419,

35064 Rennes cedex. 


\section{1 - INTRODUCTION}

The Patients suffering from Postural Deficiency Syndrome (PDS) have pains or present balance disorders. Their pains are generally not easily apprehended by the medical and paramedical profession. These musculoskeletal pains remain not well systematized and are secondary to a functional defect of the neurosensory system of balance. The differential diagnosis is critical and is associated to stabilometric records that testify balance disorders. The medical care of these patients

\section{2 - THE INVESTIGATORS}

This study brought together the practitioners of the Pain Center of the University Hospital of Rennes, the Podiatry and Chiropodist Institute of Rennes, a specialist multidisciplinary

\section{3 - THE STUDIED POPULATION}

18 patients suffering from Postural Deficiency Syndrome (PDS) were compared to a reference population, comprising 55 chronic pain patients (for more than 2 months).

Among the 18 PDS patients, we distinguished two different subpopulations:

- The first group comprised patients examined at the Podiatry and Chiropodist Institute of Rennes that presented average symptoms; requires a specialist multidisciplinary team to define a treatment strategy and then a postural stabilization with adapted musculoskeletal upgrading and sensory stimulation. We assess the psychological pain of the postural patients versus a reference population comprising chronic pain patients. Our objective is to define this psychological pain and find the most adapted tool to highlight this pain that would be easy to use by all practitioners interested in this population.

office and the National Institute of Health at the University of Rennes 1.

The tests were conducted by the clinical psychologist, Angélique Jorand.
- A second group comprising the patients of a specialist's practice suffering from more severe PDS.

The chronic pain patients who were examined to constitute the reference population were recruited at the Pain Centre of the University Hospital of Rennes and at the Podiatry and Chiropodist Institute of Rennes. 
4 - METHODOLOGY

\section{4 - 1 - Study ouline}

This is a semi-open multicentre retrospective study.

\section{4 - 2 - Calculation of the required number of patients}

The number of patients required for a reliable statistical calculation is determined by a series of interim analysis. We stopped integrating subjects once we obtained steady factor analysis (the ratio of correlation between the factors obtained on $n$ first subjects and the analysis of the following $n+p$ of the order of 0,90 ).

\section{4 - 3 - Schedule}

Beginning of inclusion: october 2005. End of inclusion: december 2005.

\section{5 - STATISTICAL METHOD}

We chose not to use scores for each scale but correspondence factor analysis that helped us conduct a multidimensional evaluation. This method of calculation helps to make a connection between the individuals who got similar test results.

6 - RESULTS

\section{6 - 1 - Main criteria: Anxiety Depression Score}

This score concerns items in relation with slight anxiety or depression. It is simple and can be self-evaluated.

\section{4 - 4 - Evaluation tools}

\section{4 - 4 - 1 - The main evaluation criteria: anxietydepression score (annexe 1$)^{8}$}

This score concerns items in relation with slight anxiety or depression. It is simple and can be self-evaluated.

\section{4 - 4 - 2 - Secondary criteria of this study}

- Analogical visual scales measuring the immediate pain intensity (during the test), the average pain intensity in the last 8 days and the most powerful pain in the last 8 days.

- Pain Sites Scoring 2,5,6,7.

- DALLAS multidimensional questionnaire, evaluating the effect on life of patients ${ }^{3,4}$.

- Abridgement Beck Questionnaire evaluating depression ${ }^{1}$.

All questions of the test are simultaneously analyzed and the breakdown of population, according to certain criteria, enables to better define the population studied.

6 - 1 - 1 - The values were broken down in two distinct populations designated as "anxious or depressed" (41 patients, of whom 30 are chronic pain and 11 postural) and as "not anxious or depressed" (27 patients, of whom 23 are chronic pain and 4 postural). 
6 - 1 - 2 - The khi2 test was conducted but did not reveal significant differences between the 2 populations.

However, we observed a large population of postural patients who suffered from anxiety and moderate depression, corresponding to our clinical impression. It has to be noted that the profile of postural patients is similar to that of chronic pain patients on the psychological plan.

Thanks to this test, the practitioner should refer his patient to specialized medical care adapted to his case.

\section{6 - 2 - Pain intensity}

The pain intensity was measured through analogical visual scales that are simple tools usually used in hospitals.

The analogical visual scales measured: the immediate pain intensity during the test, the average pain intensity in the last 8 days and the most powerful pain in the last 8 days.

According to these criteria, the patients suffering from chronic pain expressed much higher pain than the postural patients.

\section{6 - 3 - Pain Sites Scoring}

6 - 3 - 1 - The values go from 0 to 5 for a maximum score of 38 , corresponding to 38 predefined zones on the diagram representing the whole body from behind and from the front.

\section{6 - 3 - 2 - Theses values are distributed as follows:}

- Value 0: 17 patients

- Value 1: 35 patients

- Value 2: 13 patients

- Value 3: 5 patients

- Value 4: 2 patients

- Value 5: 1 patient
These are low, grouped and non contributory values used to differentiate the populations.

We were surprised by these values because this test is known to be well correlated with psychological tests. We believe that the use of this test during medical care is less relevant than during the initial recruitment phase when the pain is the highest. A study of a prospective population at the beginning of the treatment would be required before discarding this test, which seems interesting and easy to use. It is a good intermediate in the discussion between the practitioner and the patient.

The patients with high values at the topographic score $(3,4$ and 5$)$ also show results at the Beck questionnaire that reveal a clear depression. But the low population does not allow us to assess it statistically.

\section{6 - 4 - Dallas questionnaire analysis}

The Dallas multidimensional questionnaire evaluates the effect of pain on life of patients.

The results are clearly partitioned because most patients chose extreme values to qualify their pain. We can therefore differentiate patients for whom pain has a strong effect on their daily life from other patients with low effect.

6 - 4 - 1 - For severe statistical criteria: the patients who chose maximum values (19 patients with strong effect) comprise 18 chronic pain and 1 postural patient. The group of patients with lower values (21 with low effect) comprises 12 chronic pain and 9 postural patients.

Notion of severe statistical criteria (contribution to the characteristic value of the factor): 
This notion corresponds to the number of positive answers multiplied by the square of coordinates of the considered point in a correspondence factor analysis. A tested patient participates all the more to the definition of the evaluation criteria that the point representing him is far from the rest of the tested population.

6 - 4 - 2 - For less severe statistical criteria: 42 suffering patients of whom 38 are chronic pain and 4 postural, whereas 34 are low pain patients (21 chronic pain and 13 postural).

\section{6 - 4 - 3 - The khi2 test was conducted on the studied population with less severe criteria}

\begin{tabular}{|l|c|c|}
\hline khi2 $=8.92$ for $p<0,0001$ & Pain + & Pain - \\
\hline Chronic pain patients & 38 & 21 \\
\hline Postural patients & 4 & 13 \\
\hline
\end{tabular}

The calculation of khi2 reveals a highly significant result (khi2 $=8.92$ for $p<0,0001$ ).

Results are summarized in the following tables:
This test shows the effect of pain on daily life (daily activities, work and leisure, anxiety and depression, social life). The significant difference between these two populations reveals the small infirmity of postural patients on usual items of chronic pain patients and the need to use a more specific scale revealing their functional problems.

\section{6 - 5 - Beck questionnaire analysis}

The abridgement Beck Questionnaire evaluates depression.

6 - 5 - 1 - The population is divided into two main subpopulations: the depressed patients and the undepressed patients. This is less obvious than above and an intermediate population will need to be studied subsequently. This test evaluates the severity of the psychological pain on the depressive slope. This test shows the importance of a specialized treatment in case of positive results. The questions raised by this test require specialists (psychologists, psychiatrists...).

\begin{tabular}{|l|c|c|c|}
\hline DALLAS & \multicolumn{2}{|c|}{} \\
\hline Severe criteria & 40 patients studied & Strong effect & Moderate effect \\
\hline Chronic pain & 30 & 18 & 12 \\
\hline Postural patients & 10 & 1 & 9 \\
\hline
\end{tabular}

\begin{tabular}{|l|c|c|c|}
\hline DALLAS & \multicolumn{3}{|c|}{} \\
\hline Less severe criteria & 76 patients studied & Effect + & Effect + - \\
\hline Chronic pain & 59 & 38 & 21 \\
\hline Postural patients & 17 & 4 & 13 \\
\hline
\end{tabular}




\section{6 - 5 - 2 - To date the two subpopulations are as follows:}

\section{- Depressed patients (test with very si gnificant score):}

- severe criteria: 15 patients of whom 14 are chronic pain and 1 postural patient;

- less severe criteria: 28 patients of whom 22 are chronic pain and 6 postural patients.
- "Undepressed" patients (selection of items not showing a depressive ground but other problems):

- severe criteria: 11 patients of whom 8 are chronic pain and 3 postural patients;

- less severe criteria: 46 patients of whom 35 are chronic pain and 11 postural patients.

These results are summarized in the following table:

\begin{tabular}{|l|c|c|c|c|}
\hline & \multicolumn{2}{|c|}{ "Depressed» } & \multicolumn{2}{c|}{ "Undepressed» } \\
\hline Statistical criteria & Severe criteria & Less severe criteria & Severe criteria & Less severe criteria \\
\hline Chronic pain & 14 & 22 & 8 & 35 \\
\hline Postural patients & 1 & 6 & 3 & 11 \\
\hline
\end{tabular}

6 - 5 - 3 - The khi2 test does not show significant differences between chronic pain and postural patients.

\section{CONCLUSION}

The Patients suffering from Postural Deficiency Syndrome (PDS) are typical chronic pain patients. They suffer less on the functional plan but they present real psychological suffering.

This psychological suffering requires an adapted care and the use of a simple and discriminant tool.

The tests used in this study are the abridgement Beck Questionnaire, Anxiety Depression Score, the Dallas questionnaire and visual topographic Score.

The Beck questionnaire, international reference, is not adapted to our population. It is indeed not easy for non psychotherapy specialists to use this questionnaire. The Dallas questionnaire enables to measure functional problems more than the psychological pain. Daily functional problems seem less severe for PDS patients than for chronic pain patients. This test is therefore not much adapted to PDS patients. In our opinion, the visual topographical score, not much informative in this study, remains an interesting tool between the patient and the practitioners during medical care, prospectively.

The Anxiety Depression Score, main criteria of our study, is very well correlated to the Beck score and can be used alone for PDS patients. It helps non psychologists to distinguish PDS patients 
requiring adapted treatment. Secondary psychological pain with chronic symp- toms indeed acts as a break in the medical care of PDS patients.

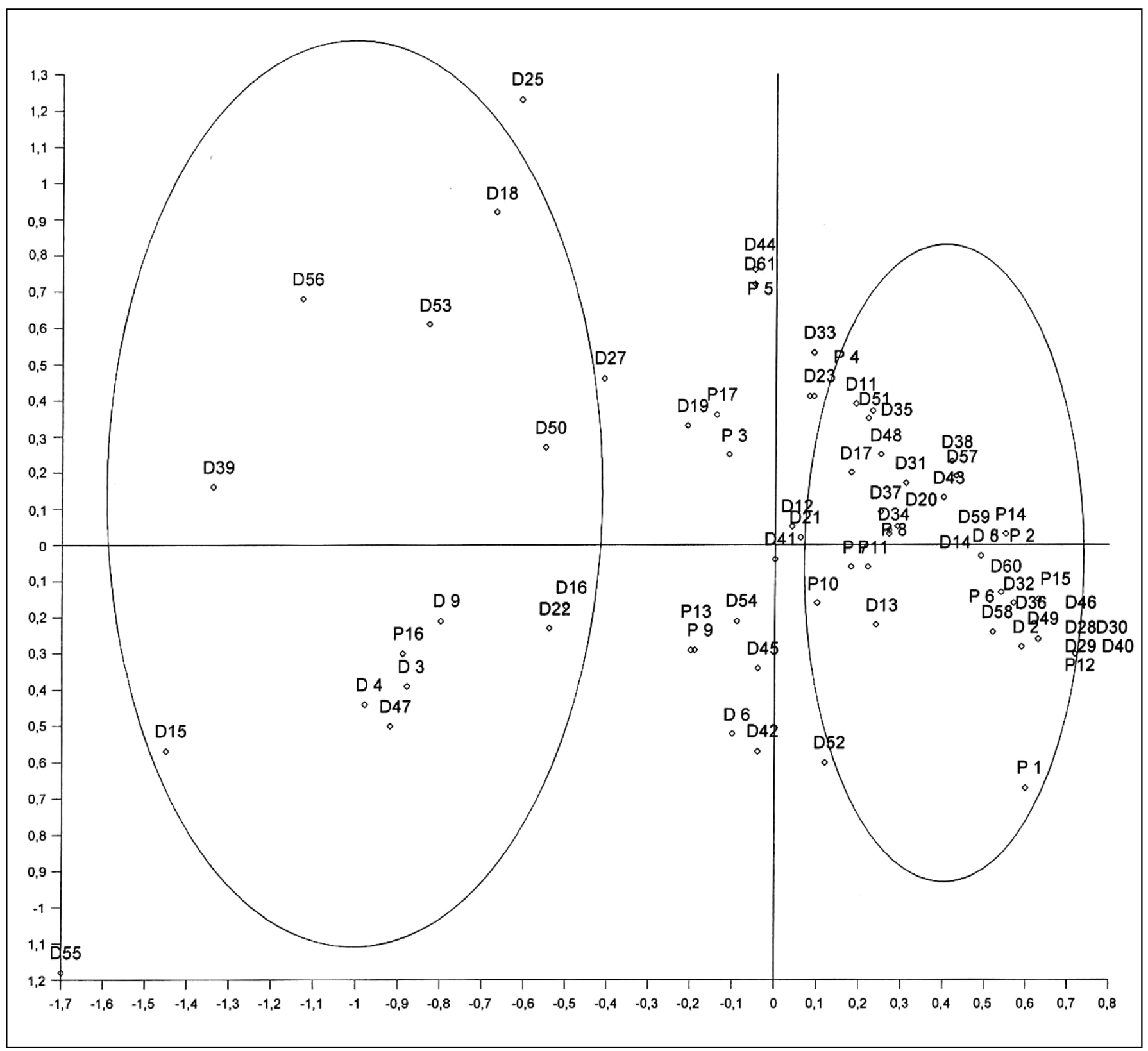

Fac simile represents a projection according to 2 main items of the matching factor analysis of the Beck Questionnaire. This projection isolates 2 subgroups: the depressed patients on the left of the ordinate axis and the undepressed on the right of this axis. 


\section{REFERENCES}

1. Atger T et al. Validation Study of the Depressive Experience Questionnaire. Encephale 2003- sept-oct;29(5):445-55.

2. Chan CW, Goldman D, Ilstrup DM, Kunselman AR, O'Neill PI. The Pain Drawing and Waddel's Nonoraganic Physical Signs in Chronic Low Back Pain. Spine, Vol. 18, Number 13, pp. 1717-1722.

3. Deyo RA, Anderson G, Bombardier C, Cherkin DC, Keller RB, Lee CK, Liang MH, Liang B, Libscomb B, P. She Spratt KF, Weinstein I.IN. Outcom Measures for Studying Patients With Low Back Pain. Spine, Vol. 19, N¹8S, pp. 2032S-2336S.

4. Deyo RA, Centor RM. Assessing the Responsiveness of Functional Scales to Clinical Change: An Analogy to Diagnostic Test Performance. Journal of chronic Disease, 1986, Vol. 39, N 11, pp. 897-906.

5. Öhlund C, Eek Claes, Palmblad S, Areskoug B, Nachemson A. Quantified Pain Drawing in Subacute Low Back Pain. Spine, 1996, Vol. 21, N 9, pp. 1021-1031.

6. Parker H, Wood PLR, Mainc J. The Use of Pain Drawing As a Screening Measure to Predict Psychological Distress in Chronic Low Back Pain. Spine. Vol. 20, № 2, pp. 236243.

7. Ransford AO, Cairns D, Mooney V. The Pain Drawing as an Aid to the Psychologic Evaluation of Pathients With Low-back Pain. Spine, June 1976, Volume 1, N² 2.

8. Wiltse LL, Rochio PD. Preoperative Psychological Tests as Predicators of Success of Chemonucleolysis in the Treatment of Low-Back Syndrome. Journal of Bone and Joint Surgery, 1975, 57-A; 478-83. 